\title{
Evaluación y análisis del índice de severidad tomográfico y clasificación de Atlanta 2012 en pancreatitis aguda severa
}

\author{
Evaluation and analysis of the tomographic severity index and Atlanta 2012 \\ classification in patients with severe acute pancreatitis
}

\author{
Braulio Giovanni Velásquez-Cuasquén ${ }^{1} \mathbb{D}$, German Hernando Ruiz-Beltrán² ${ }^{2}$, \\ Claudia Milena Orozco-Chamorro ${ }^{3} \mathbb{D}$, Jesús Eduardo Díaz-Realpe $\mathbb{D}^{4}$, Lía Jasmín Jiménez $\mathbb{1}$, \\ Daniel Alejandro Fernández-Bolaños ${ }^{1} \mathbb{D}$, Ángela María Merchán-Galvis ${ }^{5} \mathbb{D}$
}

1 Médico, residente de Cirugía general, Universidad del Cauca, Popayán, Colombia.

2 Médico, especialista en Radiología intervencionista, Hospital Universitario San José, Departamento de Medicina Interna, Universidad del Cauca, Popayán, Colombia.

3 Médica, Hospital Susana López de Valencia, Popayán, Colombia.

4 Médico, especialista en Cirugía general y en Gastroenterología, Hospital Universitario San José, Departamento de Ciencias Quirúrgicas, Universidad del Cauca, Popayán, Colombia.

5 Médica, MSc en Epidemiología clínica, profesora Departamento de Medicina Social y Salud Familiar, Universidad del Cauca, Popayán, Colombia.

\section{Resumen}

Introducción. En el paciente con pancreatitis aguda severa, la presencia de necrosis infectada y falla multiorgánica se asocian con una mortalidad del 20-40 \%. La tomografía computarizada con contraste intravenoso y la clasificación del Consenso de Atlanta 2012 son importantes herramientas de diagnóstico para el tratamiento oportuno. En esta investigación, se analizó la relación del índice de severidad tomográfico y los cambios morfológicos locales según dicha clasificación, con la estancia hospitalaria, intervención, infección y mortalidad de los pacientes.

Métodos. Estudio de cohorte retrospectiva realizado entre los años 2015 y 2019, donde se incluyeron pacientes mayores de 15 años con pancreatitis aguda severa diagnosticado por tomografía computarizada con contraste, y se evaluó el índice de severidad tomográfico y los cambios morfológicos según la clasificación de Atlanta 2012, en relación con los desenlaces clínicos de los pacientes.

Resultados. Se incluyeron 56 pacientes, en el 82,1\% $(n=46)$ de los casos la causa fue litiásica. La falla orgánica fue principalmente pulmonar 53,6 \% (n=30) y cardiovascular 55,4 \% (n=31). Según la tomografía, se clasificó como severa (7-10 puntos) en el 91,1\% $(n=51)$ de los pacientes. En pacientes con necrosis amurallada infectada

Fecha de recibido: 15/09/2020 - Fecha de aceptación: 25/01/2021 - Fecha de publicación en línea: 19/04/2021

Correspondencia: Braulio G. Velásquez-Cuasquén, Carrera 6 \# 13 N- 50, Departamento de Ciencias Quirúrgicas, Facultad de Ciencias de la Salud, Universidad del Cauca, Popayán, Colombia. Teléfono: +57 2 8209870. Correo electrónico: giovannyv@unicauca.edu.co Citar como: Velásquez-Cuasquén BG, Orozco-Chamorro CM, Ruiz-Beltrán GH, Jiménez-Ramírez LJ, et al. Evaluación y análisis del índice de severidad tomográfico y clasificación de Atlanta 2012 en pacientes con pancreatitis aguda severa. Rev Colomb Cir. 2021;36:471-80. https://doi.org/10.30944/20117582.834

Este es un artículo de acceso abierto bajo una Licencia Creative Commons - BY-NC-ND https://creativecommons.org/licenses/by-ncnd/4.0/deed.es 
la estancia hospitalaria media fue mayor (78,5 días); en todos los pacientes con pancreatitis severa se encontró infección y fueron sometidos a algún tipo de intervención. La mortalidad fue menor del $10 \%$ (n=5).

Discusión. El índice de severidad tomográfica para la categorización de severo se correlacionó en un 90 \% con pancreatitis aguda severa. Una tomografía de control a la cuarta semana podría identificar complicaciones tardías para un manejo precoz.

Palabras clave: pancreatitis; necrosis; infección; tomografía; mortalidad.

\begin{abstract}
Introduction. In patients with severe acute pancreatitis, the presence of infected necrosis and multiple organ failure are associated with a mortality of 20-40\%. Computed tomography with intravenous contrast and the 2012 Atlanta Consensus classification are important diagnostic tools for timely treatment. In this research, the relationship between the tomographic severity index and the local morphological changes according to that classification, with the hospital stay, intervention, infection and mortality of the patients was analyzed.

Methods. Retrospective cohort study carried out between the years 2015 and 2019, which included patients older than 15 years with severe acute pancreatitis diagnosed by contrast computed tomography, the tomographic severity index and morphological changes according to the Atlanta 2012 classification were evaluated, in relationship with the clinical outcomes of the patients.

Results. Fifty-six patients were included, in $82.1 \%(\mathrm{n}=46)$ of the cases the cause was lithiasis. Organ failure was mainly pulmonary $53.6 \%(n=30)$ and cardiovascular $55.4 \%(n=31)$. According to the tomography, it was classified as severe (7-10) in $91.1 \%(n=51)$ of the patients. In patients with infected walled necrosis, the mean hospital stay was longer (78.5 days); infection was found in all patients with severe pancreatitis and they underwent some type of intervention. Mortality was less than $10 \%(n=5)$.
\end{abstract}

Discussion. The tomographic severity index for the categorization of severity is $90 \%$ correlated with severe acute pancreatitis. A control tomography at the fourth week could identify late complications for early management.

Keywords: pancreatitis; complications; necrosis; infection; tomography; severity of illness index; mortality.

\section{Introducción}

La pancreatitis aguda (PA) se genera por la activación no regulada de la tripsina dentro de las células acinares pancreáticas, desencadenando una presentación clínica variable, desde una injuria local, síndrome de respuesta inflamatoria sistémica, falla orgánica e incluso la muerte ${ }^{1}$. Esta entidad constituye una situación de emergencia abdominal y una causa importante de hospitalización, en donde la evolución, el manejo y el diagnostico se convierten en un reto importante para el clínico ${ }^{2}$. Las principales causas de PA están directamente relacionadas con la patología biliar y el consumo de alcohol, pero también se ha encontrado relación con dislipidemia, uso de algunos fármacos y procesos infecciosos ${ }^{3}$.
La incidencia de pancreatitis aguda a nivel mundial ha ido en aumento, oscilando de 4,9 a 73,4 por 100,000 personas ${ }^{1,4}$, y en Latinoamérica varia en los diferentes países ${ }^{5,6}$. En Colombia, la incidencia de esta enfermedad no está muy bien determinada, pero se cree que es de 35 a 80 casos por 100,000 habitantes ${ }^{7}$. En el departamento de Cauca, se cuenta con pocos estudios al respecto; en un estudio en donde se revisaron retrospectivamente 162 historias clínicas entre el año 2000 y 2005, se encontró el diagnostico de pancreatitis aguda severa (PAS) en un $25 \%$ de los pacientes ${ }^{8}$.

Dentro de los factores que se asocian con peor pronóstico se han identificado las complicaciones infecciosas, la necrosis pancreática y la falla 
orgánica múltiple, las cuales pueden asociarse con una mortalidad hasta del $40 \%{ }^{9}$.

La tomografía computarizada (TC) con contraste intravenoso, es una herramienta determinante para la identificación de estas situaciones, siendo de gran utilidad para realizar el diagnóstico, reconocer complicaciones y evaluar el grado de inflamación y necrosis, estableciendo un pronóstico de severidad de la enfermedad ${ }^{4}$.

La introducción del índice de severidad de la TC, en 1994, representó un avance significativo en la evaluación de los pacientes con PA. Este índice aceptado internacionalmente, se basa en la evaluación de la presencia y el grado de inflamación y necrosis pancreática, y no solo permite diferenciar con precisión la pancreatitis leve de la severa, sino que también se correlaciona numéricamente con el pronóstico del paciente ${ }^{10}$. Sin embargo, a pesar de que el índice de gravedad de la TC se ha utilizado con éxito para predecir la morbilidad y la mortalidad en pacientes con PA, la literatura reciente ha revelado limitaciones de este índice sugiriendo que no correlaciona el estado clínico del paciente con el compromiso morfológico de las lesiones pancreáticas ${ }^{11}$.

Esta falencia en el pronóstico de la enfermedad y estandarización de las lesiones mejoró con el Consenso Internacional de Atlanta $2012^{12}$, el cual actualmente es el sistema más utilizado para clasificar la PA en leve, moderada o severa, y permite además identificar dos fases, la temprana y la tardía ${ }^{9}$. La forma severa de la pancreatitis generalmente se presenta con insuficiencia orgánica persistente por más de 48 horas, complicaciones locales y sistémicas, que pueden empeorar si se asocian con necrosis infectada ${ }^{13}$.

El Consenso también describe los cambios morfológicos asociados con la necrosis, entre ellas la colección necrótica aguda (ANC, por sus siglas en inglés) y la necrosis amurallada (WON, por sus siglas en inglés). La ANC es una colección que se observa durante las primeras cuatro semanas y que contiene una cantidad variable de líquido y tejido necrótico que involucra el parénquima pan- creático o los tejidos peripancreáticos. La WON es una colección encapsulada y madura de necrosis pancreática o peripancreática con una pared inflamatoria bien definida y realzada, cuya maduración suele tardar 4 semanas o más después del inicio de la pancreatitis aguda ${ }^{12,13}$.

El objetivo de este estudio fue correlacionar el índice de severidad tomográfico y los cambios morfológicos locales según la clasificación de Atlanta 2012 con la estancia hospitalaria, la necesidad de cirugía, presencia de infección y mortalidad de los pacientes.

\section{Métodos}

Se llevó a cabo un estudio observacional, tipo cohorte retrospectiva, en el período comprendido entre el $1^{\circ}$ de enero de 2015 y el 31 de diciembre de 2019, en el Hospital Universitario San José de Popayán, Colombia. Se incluyeron todos los pacientes mayores de 15 años con diagnóstico establecido de pancreatitis aguda severa a quienes se les realizó un estudio tomográfico de abdomen utilizando un equipo Siemens ${ }^{\circledR}$ multidetector helicoidal de 64 cortes, en fase simple, arterial y venosa. Se excluyeron los pacientes que no completaron el tiempo de seguimiento hasta el egreso (remisión o alta voluntaria).

Los datos demográficos y las demás variables clínicas se obtuvieron de la historia clínica electrónica. Las imágenes tomográficas fueron revisadas por un radiólogo experto. La severidad de la pancreatitis para cada caso fue evaluada utilizando el índice de severidad de la TC desarrollado por Balthazar et al. ${ }^{14} \mathrm{y}$ los cambios morfológicos locales según el Consenso de Atlanta $2012^{12}$.

La información se registró en la plataforma de cuestionarios electrónicos Clinapsis ${ }^{\circledR}$. Para el análisis estadístico se utilizó el programa SPSS ${ }^{\text {тм }}$ versión 25 , empleando para las variables cualitativas, frecuencias y proporciones, así como medidas de tendencia central y de dispersión para las variables cuantitativas. Se hizo un análisis bivariado para establecer la correlación entre el índice tomográfico, la clasificación de Atlanta 2012 y los desenlaces de los pacientes. 


\section{Resultados}

Se revisaron 223 historias clínicas, de las cuales 87 pacientes tuvieron PAS y 56 cumplieron con los criterios, incluida la realización de TC (figura 1). La edad media al momento del diagnóstico fue de 53,5 años (DE 20,9). El 51,8 \% (n=29) de los pacientes fueron de sexo masculino. La principal causa de pancreatitis correspondió a la litiásica en $82,1 \%(n=46)$, seguida por la dislipidemia en 12,5\% (n=7) de los casos. El 7,1\% $(n=4)$ de los pacientes presentaron PAS posterior a la realización de colangiopancreatografía retrógrada endoscópica (CPRE) (tabla 1).

Los sistemas afectados en el paciente con falla orgánica fueron principalmente el pulmonar en $53,6 \%(n=30)$ y el cardiovascular en el $55,4 \%$ $(\mathrm{n}=31)$. El promedio de tiempo para la detección de la falla orgánica fue de 3,1 días (DE 2,3), con un
Tabla 1. Características sociodemográficas de los pacientes con pancreatitis aguda severa diagnosticados por tomografía computarizada con contraste.

\begin{tabular}{llcc}
\hline & & \multicolumn{2}{c}{ Total } \\
\cline { 3 - 4 } Sexo & $\mathbf{n}$ & $\%$ \\
\hline \multirow{2}{*}{ Edad } & Hombre & 29 & 51,8 \\
& Mujer & 27 & 48,2 \\
\hline \multirow{6}{*}{ Causa } & Media & & 53,5 \\
& DE & & 20,9 \\
& Litiasis & 46 & 82,1 \\
& Dislipidemia & 7 & 12,5 \\
& Idiopática & 5 & 8,9 \\
& Clcohólica & 4 & 7,1 \\
& CPRE & 4 & 7,1 \\
& Otras & 3 & 5,4 \\
& Trauma & 1 & 1,8 \\
\hline
\end{tabular}

*DE: desviación estándar; CPRE: colangiopancreatografía retrógrada endoscópica.

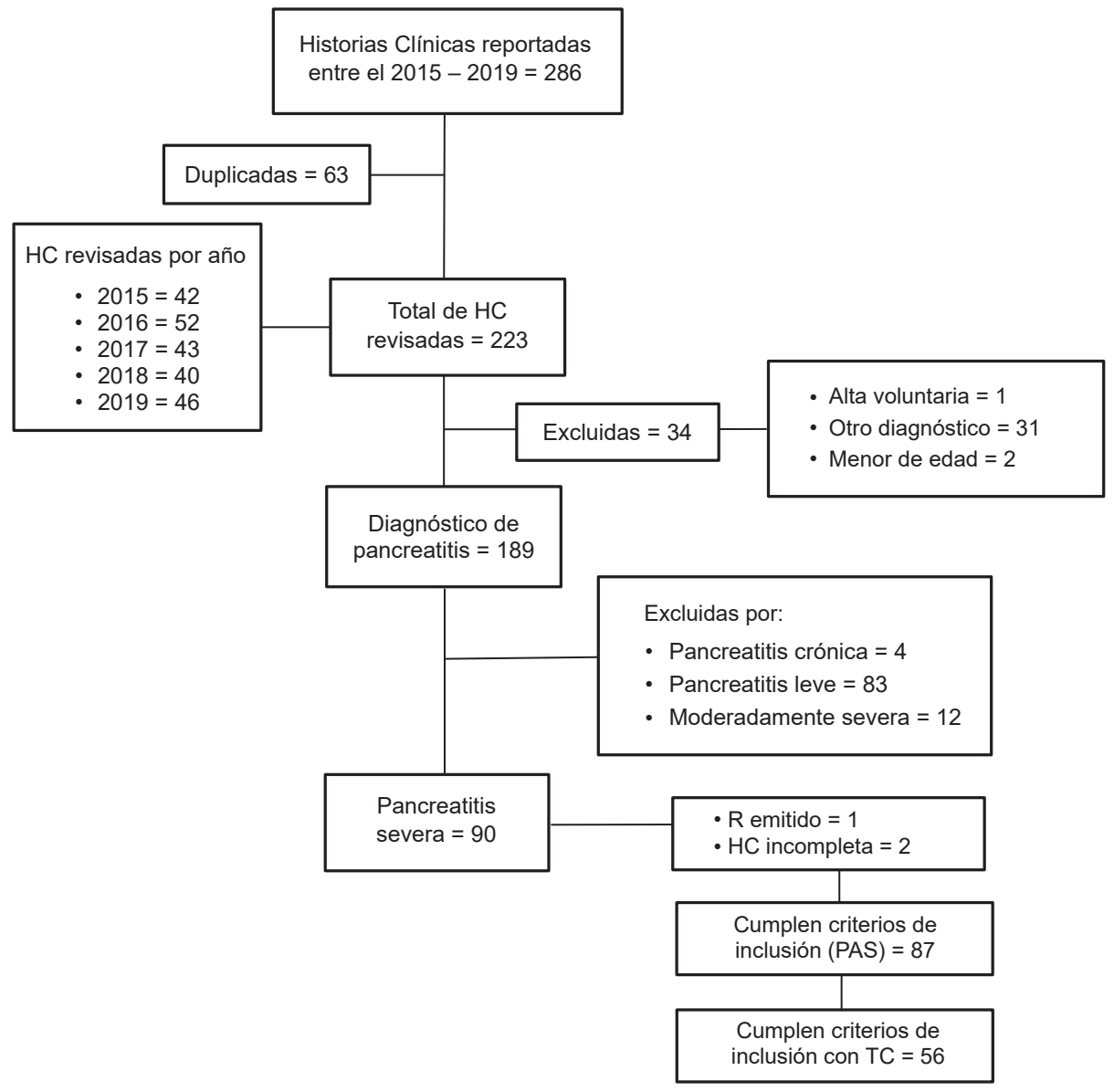

HC: historia clínica; PAS: pancreatitis aguda severa; TC: tomografía computarizada.

Figura 1. Flujograma de selección de pacientes con pancreatitis severa 
puntaje de Marshall promedio de 2,2 (tabla 2). De acuerdo con la tomografía, se encontró que el 44,6 \% (n=25) de los pacientes tenían clasificación de Balthazar C y el 55,4\% $(n=31)$ no presentaban necrosis, de esta forma se calculó el índice de severidad tomográfica, siendo de 7-10 puntos en el 91,1\% ( $\mathrm{n}=51$ ) de los pacientes (tabla 3$)$.

Con base en la clasificación de Atlanta 2012, se realizó la revisión tomográfica para determinar las complicaciones pancreáticas locales desarrolladas antes y después de la cuarta semana. Se encontró una colección peripancreática fluida aguda (APFC, por sus siglas en inglés) en el 46,4\% $(\mathrm{n}=26)$ de los pacientes, con un tiempo medio de aparición de 4,3 días; colección necrótica aguda estéril (ANCe, por sus siglas en inglés) en el 37,3 \% ( $\mathrm{n}=22)$, con un tiempo medio de aparición de 5,1 días; colección necrótica aguda infectada (ANCi, por sus siglas en inglés) en el 8,92 \% ( $n=5)$ de los casos, con un tiempo de aparición de 15 días, y necrosis amurallada (WON) en el 18,6\% $(n=11)$, después de 47 días. Al analizar la evolución de las lesiones estériles hacia infectadas, se encontró que dos pacientes con APFC progresaron a WON. Cuatro con ANCe progresaron a ANCi y siete a WON, cambios que no fueron estadísticamente significativos ( $\mathrm{p}=0,051 \mathrm{y} \mathrm{p}=0,065$ respectivamente) (tabla 4).

Del total de pacientes, el 73,2 \% (n=41) fueron llevados a cirugía; $31,7 \%(n=13)$ por vía laparoscópica; por medio de abordaje percutáneo el 48,8 \% (n=20) y mediante cirugía abierta el $34,1 \%(n=14)$. Se realizó CPRE al 46,2 \% ( $n=24)$ de los pacientes. El $22 \%$ (n=9) tuvieron abdomen abierto con un promedio de 28,3 días. Con respecto al soporte metabólico, el 46,4\% (n=26) recibieron nutrición enteral, 21,4 \% (n=12) pa-
Tabla 2. Falla orgánica ocurrida en los pacientes con pancreatitis aguda severa.

\begin{tabular}{lcc}
\hline \multirow{2}{*}{ Falla orgánica } & \multicolumn{2}{c}{ Total } \\
\cline { 2 - 3 } Falla cardiovascular & $\mathbf{n}$ & $\%$ \\
Falla pulmonar & 31 & 55,4 \\
Falla renal & 30 & 53,6 \\
Falla hepática & 14 & 25,0 \\
Falla del sistema nervioso central & 6 & 10,7 \\
\hline Aparición en días & 1 & 1,8 \\
\hline Media & & \\
Desviación estándar & & 3,1 \\
Mínima-máxima & & 2,3 \\
\hline Puntaje de Severidad - Marshall & & \\
\hline Media & & 2,14 \\
Desviación estándar & & 1,2 \\
Mínima-máxima & & $1-6$ \\
\hline
\end{tabular}

Tabla 3. Índice de severidad tomográfico en los pacientes con pancreatitis aguda.

\begin{tabular}{lccc}
\hline Criterio & & $\mathbf{n}$ & $\%$ \\
\hline \multirow{3}{*}{ Balthazar } & A & 5 & 8,9 \\
& C & 6 & 10,7 \\
& D & 25 & 44,6 \\
Necrosis & E & 10 & 17,9 \\
& Menor del 30 \% & 9 & 17,9 \\
& De 30-50 \% & 8 & $10,4,4$ \\
Severidad & Mayor del 50 \% & 8 & 14,3 \\
& Menor de 3 puntos & 5 & 8,9 \\
& 7-10 puntos & 51 & 91,1 \\
\hline
\end{tabular}

Tabla 4. Complicaciones pancreáticas locales estériles e infectadas

\begin{tabular}{|c|c|c|c|c|c|c|c|}
\hline & & \multicolumn{6}{|c|}{ Clasificación de Atlanta 2012} \\
\hline & & \multicolumn{3}{|c|}{ ANC Infectada } & \multicolumn{3}{|c|}{ WON } \\
\hline & & Si & No & $\mathbf{p}$ & Si & No & $\mathbf{p}$ \\
\hline \multirow{2}{*}{ APFC } & $\mathrm{Si}$ & 0 & 26 & \multirow{2}{*}{0,029} & 2 & 24 & \multirow{2}{*}{0,036} \\
\hline & No & 5 & 25 & & 9 & 21 & \\
\hline \multirow{2}{*}{ ANC estéril } & $\mathrm{Si}$ & 4 & 18 & \multirow{2}{*}{0,051} & 7 & 15 & \multirow{2}{*}{0,065} \\
\hline & No & 1 & 33 & & 4 & 30 & \\
\hline
\end{tabular}

APFC: colección peripancreática fluida aguda; ANC: colección necrótica aguda; WON: necrosis amurallada. 
renteral y mixta un 32,1\% ( $\mathrm{n}=18)$. La mortalidad ocurrió en el 3,6 \% (n=2), y en ambos pacientes estuvo asociada a complicaciones pancreáticas (100\%) (tabla 5).

\section{Discusión}

A diferencia de lo descrito por otros reportes en donde predomina el sexo femenino ${ }^{1,7,13}$, en el presente estudio la mayoría de los pacientes fueron hombres, lo que puede ser explicado por mecanismos inmunológicos y genéticos asociados con la disminución del umbral de la activación de la tripsina, los cuales se ven de manera más frecuente en el sexo masculino ${ }^{14}$. Acorde a la literatura mundial ${ }^{1,15,16}$ y a un estudio local ${ }^{8}$, se encontró que la principal causa de pancreatitis fue la litiásica. Se ha descrito que la pancreatitis aguda aumenta con la edad ${ }^{1}$, correlacionándose con un mayor riesgo de severidad y complicaciones de predominio en el sexo masculino ${ }^{17}$; la edad media de los pacientes de este estudio fue de 53,5 años (DE 20,9).

La clasificación de la severidad se realizó utilizando como parámetro la escala de APACHE II mayor a 8 y el Consenso de Atlanta 2012, encontrando que cerca de la mitad de los pacientes pre- sentaron pancreatitis aguda severa, cifra superior a la informada en la literatura mundial que tan solo menciona el $20 \%{ }^{18}$. Esto puede ser explicado porque el Hospital Universitario San José, de Popayán, es centro de referencia en la región y recibe la mayoría de pacientes con esta patología, especialmente aquellos con indicación de manejo en unidad de cuidado intensivo.

Con respecto a la falla orgánica, en este estudio la disfunción pulmonar y el compromiso cardiovascular se presentaron en más del $50 \%$ de los pacientes con un tiempo medio de aparición de 3 días, aunque algunos autores describen el compromiso renal como el principal sistema afectado ${ }^{18}$. El mayor o menor compromiso de los diferentes órganos, además del tiempo de aparición en cada uno de ellos puede verse afectado por la heterogeneidad de los pacientes y el grado de infección y necrosis asociada ${ }^{19}$. En la evaluación imagenológica, el $90 \%$ de los pacientes presentó un índice de severidad tomográfico entre 7-10 puntos, acorde al tipo de pacientes incluidos específicamente con pancreatitis aguda severa.

Para los desenlaces como estancia hospitalaria, cirugía o infección, no se encontraron cambios

Tabla 5. Tratamiento y desenlace de los pacientes con pancreatitis aguda severa.

\begin{tabular}{llcc}
\hline & & $\mathbf{n}$ & $\%$ \\
\hline Colangiopancreatografía retrograda endoscópica & 24 & 46,2 \\
\hline \multirow{2}{*}{ Cirugía } & No & 15 & 26,8 \\
& $\mathrm{Si}$ & 41 & 73,2 \\
\hline \multirow{2}{*}{ Tipo de cirugía } & Abierta & 14 & 34,1 \\
& Drenaje percutáneo & 20 & 48,8 \\
& Laparoscópica & 13 & 31,7 \\
\hline Abdomen abierto & & 9 & 22,0 \\
\hline Tiempo en días & Media (Desviación estándar) & 28,3 & $(27,3)$ \\
\hline \multirow{2}{*}{ Tipo de nutrición } & Enteral & 26 & 46,4 \\
& Parenteral & 12 & 21,4 \\
& Mixta & 18 & 32,1 \\
\hline \multirow{2}{*}{ Desenlace } & Vivo & 54 & 96,4 \\
\hline Muertes relacionadas & No & 2 & 3,6 \\
\hline con pancreatitis & Si & 0 & 0,0 \\
\hline
\end{tabular}


estadísticamente significativos. El 8,9 \% de los pacientes correspondieron al grupo de pancreatitis leve, valor no acorde con la población estudiada, lo que sugiere limitaciones en el índice para clasificar correctamente los pacientes; esta debilidad ha sido descrita en estudios previos ${ }^{12}$, asociada en primer lugar a que las complicaciones morfológicas tanto peripancreáticas como extrapancreáticas pueden no correlacionarse con la presencia de insuficiencia orgánica, lo cual puede afectar el resultado ${ }^{16}$; y en segundo lugar, puede ser que en cinco pacientes las tomografías se tomaron en un periodo inferior a 72 horas, es decir de forma temprana, por lo que los cambios morfológicos pudieron no ser observados, clasificándose equívocamente como leves.

Según la literatura, la concordancia entre observadores para la puntuación de las TC es cercana al $75 \%{ }^{13}$, pero no hay consenso respecto a la categorización moderada usando porcentajes de necrosis de 30-50 \%. Consideramos que, en el presente estudio, esta evaluación fue más adecuada, debido a que la valoración tomográfica fue realizada por un solo radiólogo experto, quien revisó todas las tomografías para evitar el sesgo inter-observador, lo cual se ve reflejado en los resultados. No encontramos esta categoría moderada en la serie de pacientes estudiados.

Para determinar los cambios morfológicos pancreáticos locales se utilizó la revisión de Atlanta $2012{ }^{12}$, la cual estandariza la terminología en función del tiempo, permitiendo a los diferentes expertos hablar un mismo idioma. En este grupo de pacientes se tuvo en cuenta la TC inicial y los diferentes controles tomográficos, y el enfoque se dirigió hacia la evolución radiológica de las colecciones y las necrosis estériles. Se encontró que las APFC tuvieron un comportamiento inusual con respecto al descrito en el Consenso ${ }^{12}$, ya que dos de estas evolucionaron a WON en el control tomográfico. Sin embargo, la historia natural de la necrosis pancreática o peripancreática es variable, ya que puede permanecer sólida o licuarse, permanecer estéril o infectarse, persistir o desaparecer con el tiempo ${ }^{19}$.

La ANC se encontró antes de la cuarta semana, cuatro de ellas se infectaron al día 15 y requirieron intervención en el $100 \%$ de los casos, principalmente de forma percutánea como lo recomiendan los expertos ${ }^{20,21}$. Estos desenlaces fueron estadísticamente significativos y acordes con el Consenso y la guía actualizada para manejo de PAS ${ }^{10,11}$. En nuestra serie de pacientes se encontró cerca del $20 \%$ de WON, lo que se asoció con mayor estancia hospitalaria $(p=0,021)$, necesidad de intervención quirúrgica $(\mathrm{p}=0,025)$ e infección $(\mathrm{p}=0,004)$, esta última demostrada en el $100 \%$ de los casos.

Las modalidades de intervención en PAS, han cambiado con el tiempo, desde técnicas quirúrgicas abiertas y tempranas, hasta estrategias conservadoras con intervenciones luego de cuatro semanas de evolución y siguiendo un esquema desde procedimientos menos invasivos (endoscópicos o percutáneos) hasta técnicas quirúrgicas abiertas como última opción. Lo anterior ha impactado en los desenlaces asociados como el elevado estrés quirúrgico, las lesiones vasculares, fístula entero cutánea y la muerte ${ }^{13}$.

De los pacientes incluidos, $60 \%$ fueron sometidos a intervención quirúrgica, siendo la cirugía mínimamente invasiva la preferida en más del $40 \%$ de los casos, el drenaje percutáneo para manejo de colecciones se realizó en el $48 \%$, seguido de la CPRE para abordaje de las pancreatitis asociadas a colangitis, la gran mayoría de los pacientes tuvieron procedimientos combinados. Para el caso de las complicaciones pancreáticas locales, en la institución, se sigue el método "Step-up Approach" 22,23 utilizando tanto el método endoscópico como el percutáneo, lo cual se refleja en los resultados obtenidos.

Con respecto al soporte nutricional, más del $60 \%$ de los pacientes recibieron nutrición enteral con adecuada tolerancia, resultados similares a los observados en otras series y acorde a las recomendaciones actuales que indican la nutrición enteral sobre la parenteral en pacientes con PAS, debido a que disminuye las complicaciones infecciosas ${ }^{24}$.

Dentro de los factores que se asocian con peor pronóstico en los pacientes con PA se encuentran la necrosis, la infección y la falla orgánica múltiple, casos en donde se puede presentar una mortalidad variable, oscilando entre 4-7 \% para todos los casos y 20-50 \% para las PAS. 
La mortalidad estuvo asociada a complicaciones pancreáticas en la mayoría de casos, y fue del $10,6 \%$, inferior a lo descrito en la literatura mundial, pues en los mejores centros se reporta alrededor del $15 \%{ }^{21}$, una posible razón está dada por el hecho de que no se realizó tomografía en unos pacientes con PAS por la severidad de la misma, y fueron excluidos del estudio por no cumplir con los criterios de inclusión. Algunos autores describen esta falencia justificados en el estado crítico del paciente que no permite su movilización ${ }^{10}$.

Dentro de las limitaciones del presente trabajo se encuentra el carácter retrospectivo del mismo, lo que pudo generar sesgo de información, ya que en algunos pacientes los datos fueron tomados de la historia clínica de remisión.

Como fortalezas se encuentran, el número de pacientes incluidos con PAS, similar a los reportados por otros autores ${ }^{8,10,19}$, lo que permitió analizar mejor los diferentes cambios morfológicos desarrollados en el curso natural de la enfermedad.

Otra fortaleza radica en la disponibilidad de un equipo multidetector helicoidal de 64 cortes, con un buen rendimiento, sensibilidad del 97,1\% y especificidad del 98,3 \% para PAS ${ }^{25}$, y en contar con el apoyo permanente de un radiólogo experto y una lectura estandarizada, lo que disminuyó el sesgo de medición en la clasificación de los pacientes, tanto para el índice de severidad tomográfico como para el Consenso de Atlanta.

Todos los procedimientos se realizaron en la misma institución y por el mismo grupo interdisciplinario: cirugía general, cirugía minimamente invasiva, gastroenterología, radiología intervencionista, siguiendo las guías actuales de manejo y utilizando el recurso de la cirugía mínimamente invasiva, entre otros ${ }^{9}$.

\section{Conclusiones}

El índice de severidad tomográfico para la categorización de severo (7-10 puntos) se correlaciona de manera adecuada con la pancreatitis aguda severa presentada por los pacientes de este estudio. Aunque las guías no hacen una recomendación fuerte para el seguimiento radiológico, se debe estar atento a los cambios clínicos e imagenológicos antes de la cuarta semana, lo que permitirá identificar complicaciones tardías.

La presencia de WON en la TC es un indicador de mayor estancia hospitalaria, intervenciones e infección, por eso detectarla de una manera precoz podría mejorar estos desenlaces.

Futuros estudios prospectivos que evalúen los cambios morfológicos en el páncreas, teniendo en cuenta el estado clínico del paciente en función del tiempo, permitirán intervenir los desenlaces asociados en los pacientes con PAS.

\section{Cumplimiento de normas éticas}

Consentimiento informado: se contó con el aval del Comité de Ética para la Investigación del Hospital Universitario San José.

Declaración de conflictos de interés: los autores declaramos que no hay conflictos de interés

Fuentes de financiación: no se recibió ningún tipo de financiación para la realización de este trabajo.

\section{Contribución de los autores:}

Concepción y diseño del estudio: Braulio Giovanni Velásquez-Cuasquén' German Hernando Ruiz-Beltrán, Claudia Milena Orozco-Chamorro, Jesús Eduardo DíazRealpe, Lía Jasmín Jiménez, Daniel Alejandro FernándezBolaños, Ángela María Merchán-Galvis.

Adquisición de datos; Braulio Giovanni VelásquezCuasquén' German Hernando Ruiz-Beltrán, Claudia Milena Orozco-Chamorro, Jesús Eduardo Díaz-Realpe, Lía Jasmín Jiménez, Daniel Alejandro Fernández-Bolaños, Ángela María Merchán-Galvis.

Análisis e interpretación de datos: Braulio Giovanni Velásquez-Cuasquén' German Hernando Ruiz-Beltrán, Claudia Milena Orozco-Chamorro, Jesús Eduardo DíazRealpe, Lía Jasmín Jiménez, Daniel Alejandro FernándezBolaños, Ángela María Merchán-Galvis.

Redacción del manuscrito: Braulio Giovanni VelásquezCuasquén German Hernando Ruiz-Beltrán, Claudia Milena Orozco-Chamorro, Jesús Eduardo Díaz-Realpe, Lía Jasmín Jiménez, Daniel Alejandro Fernández-Bolaños, Ángela María Merchán-Galvis.

Revisión crítica: Braulio Giovanni Velásquez-Cuasquén German Hernando Ruiz-Beltrán, Claudia Milena OrozcoChamorro, Jesús Eduardo Díaz-Realpe, Lía Jasmín Jiménez, Daniel Alejandro Fernández-Bolaños, Ángela María Merchán-Galvis. 


\section{Referencias}

1. Yadav D, Lowenfels AB. The epidemiology of pancreatitis and pancreatic cancer. Gastroenterology 2013; 144:1252-61 https://doi.org/10.1053/j.gastro.2013.01.068

2. Lankisch PG, Apte M, Banks PA. Acute pancreatitis. The Lancet. 2015;386:85-96 https://doi.org/10.1016/S0140-6736(14)60649-8

3. Puerto-Horta LJ, Medina-Rojas R, Nuñez-Romero LR, Jiménez-Sánchez HC, Olaya-Ramírez JG, San Juan JF, et al. Manejo y desenlaces de la pancreatitis aguda en un hospital de cuarto nivel (Huila, Colombia), 3 años de experiencia. Rev Colomb Gastroenterol. 2019;34:10-6 https://doi.org/10.22516/25007440.243

4. Tenner S, Baillie J, Dewitt J, Vege SS. American college of gastroenterology guideline: Management of acute pancreatitis. Am J Gastroenterol. 2013;108:1400-15 https://doi.org/10.1038/ajg.2013.218

5. De Campos T, Parreira JG, Utiyama E, Rasslan S. Pesquisa nacional sobre condutas na pancreatite aguda. Rev Col Bras Cir. 2008;35:304-10 https://doi.org/10.1590/S0100-69912008000500006

6. Valdivieso-Herrera MA, Vargas-Ruiz LO, Arana-Chiang AR, Piscoya A. Situación epidemiológica de la pancreatitis aguda en Latinoamérica y alcances sobre el diagnóstico. Acta Gastroenterológica Latinoamericana. 2016;46:102-3 [fecha de acceso 14 de agosto de 2020]; disponible en: https://actagastro.org/situacion-epidemiologica-de-la-pancreatitis-aguda-en-latinoamerica-y-alcances-sobre-el-diagnostico/

7. Lipovestky F, ToneLLi C, Ramos A, Cueto G, Guimaraens $\mathrm{P}$, Reina $\mathrm{R}$, et al. Pancreatitis aguda. Su manejo en Cuidados Intensivos. Pancreat aguda. 2016;33(1) [fecha de acceso 16 de agosto de 2020]; disponible en: https://revista.sati.org.ar/index.php/MI/article/ view/461/373

8. Revelo E, Diaz J. Correlación entre la escala de apache II con la tomografía axial computarizada (TAC) y panc 3 , en pacientes con pancreatitis aguda severa en la uci del HUSJ y Clínica La Estancia durante el periodo comprendido entre enero de 2008 y junio de 2011. [Tesis de grado]. Popayán: Universidad del Cauca; 2012.

9. De Waele JJ, Rello J, Anzueto A, Moreno R, Lipman J, Sakr Y, et al. Infections and use of antibiotics in patients admitted for severe acute pancreatitis: Data from the epic II study. Surg Infect. 2014;15:132-6 https://doi.org/10.1089/sur.2012.228

10. Mortele KJ, Wiesner W, Intriere L, Shankar S, Zou KH, Kalantari BN, et al. A modified CT severity index for evaluating acute pancreatitis: Improved correlation with patient outcome. Am J Roentgenol. 2004;29:363 https://doi.org/10.1097/00006676-200411000-00140

11. Inoue $\mathrm{K}$, Hirota $\mathrm{M}$, Beppu T, Ishiko T, Kimura Y, Maeda $\mathrm{K}$, et al. Angiographic features in acute pancreatitis: The severity of abdominal vessel ischemic change reflects the severity of acute pancreatitis. J Pancreas. 2003;4:20713 (fecha de acceso: agosto 16 de 2020) disponible en: https://pubmed.ncbi.nlm.nih.gov/14614201/

12. Banks PA, Bollen TL, Dervenis C, Gooszen HG, Johnson CD, Sarr MG, et al. Classification of acute pancreatitis - 2012: Revision of the Atlanta classification and definitions by international consensus. Gut. 2013;62:102-111 https://doi.org/10.1136/gutjnl-2012-302779

13. Leppäniemi A, Tolonen M, Tarasconi A, Segovia-Lohse H, Gamberini E, Kirkpatrick AW, et al. 2019 WSES guidelines for the management of severe acute pancreatitis. World Journal of Emergency Surgery. 2019;14:1-20. https://doi.org/10.1186/s13017-019-0247-0

14. Balthazar EJ, Ranson JHC, Naidich DP, Megibow AJ, Caccavale R, Cooper MM. Acute pancreatitis: Prognostic value of CT. Radiology. 1985;156:767-772. https://doi.org/10.1148/radiology.156.3.4023241

15. Almora-Carbonell, C. Arteaga-Prado, Y, Plaza-Gonzalez, T. Prieto-Ferro, Y. Hernández-Hernández Z. Diagnóstico clínico y epidemiológico de la litiasis vesicular. Revisión bibliográfica. Rev Ciencias Médicas Pinar del Río. 2012;16:200-14. [fecha de acceso 16 de agosto de 2020]; disponible en: http://scielo.sld.cu/pdf/rpr/v16n1/rpr21112.pdf

16. Marín Ríos DF, Águila Meleros OM, Torres Ajá L, Puerto Lorenzo JA. Morbilidad y mortalidad en pacientes con pancreatitis aguda. Cienfuegos, 2014-2015. MediSur. 2019;17:210-20 [fecha de acceso 14 de agosto de 2020]; disponible en: http://scielo.sld.cu/pdf/ms/ v17n2/1727-897X-ms-17-02-210.pdf

17. Párniczky A, Kui B, Szentesi A, Balázs A, Szûcs Á, Mosztbacher D, et al. Prospective, multicenter, nationwide clinical data from 600 cases of acute pancreatitis. PLoS One. 2016;36:1-19 https://doi.org/10.1371/journal.pone.0165309

18. Surco Y, Mercado JH, Pinto J, Piscoya A, De R, Ríos L, et al. Predicción precoz de severidad en pancreatitis aguda. Rev Gastroenterol del Perú. 2012;32:241-50 [fecha de acceso 16 de agosto de 2020]; disponible en: http:// www.scielo.org.pe/scielo.php?script=sci_arttext\&pi$\mathrm{d}=\mathrm{S} 1022-51292012000300002$

19. Sahu B, Abbey P, Anand R, Kumar A, Tomer S, Malik E. Severity assessment of acute pancreatitis using CT severity index and modified CT severity index: Correlation with clinical outcomes and severity grading as per the Revised Atlanta Classification. Indian J Radiol Imaging. 2017;27:152-60 [fecha de acceso 16 de agosto de 2020]; disponible en: https://pubmed.ncbi.nlm.nih.gov/28744075/

20. Foster BR, Jensen KK, Bakis G, Shaaban AM, Coakley F V. Revised Atlanta classification for acute pancreatitis: A pictorial essay. Radiographics. 2016;36:675-87 https://doi.org/10.1148/rg.2016150097 
21. Grinsven J Van, Van Dijk SM, Dijkgraaf MG, Boermeester MA, Bollen TL, Bruno MJ, et al. Postponed or immediate drainage of infected necrotizing pancreatitis (POINTER trial): Study protocol for a randomized controlled trial. Trials. Trials 20, 239 (2019). [fecha de acceso 16 de agosto de 2020]; disponible en:

https://doi.org/10.1186/s13063-019-3315-6

22. Van Grinsven J, Van Santvoort HC, Boermeester MA, Dejong $\mathrm{CH}$, Van Eijck CH, Fockens P, et al. Timing of catheter drainage in infected necrotizing pancreatitis. Nature Reviews Gastroenterology and Hepatology. 2016;13:306-12.

https://doi.org/10.1038/nrgastro.2016.23

23. Tse F, Yuan Y. Early routine endoscopic retrograde cholangiopancreatography strategy versus early conserva- tive managementstrategyin acutegallstone pancreatitis. Cochrane Database Syst Rev. 2012;(5):CD009779. https://doi.org/10.1002/14651858.CD009779

24. Van Santvoort HC, Besselink MG, Bakker OJ, Hofker HS, Boermeester MA, Dejong CH, et al. A step-up approach or open necrosectomy for necrotizing pancreatitis. N Engl J Med. 2010;362:1491-502 [fecha de acceso 16 de agosto de 2020]; disponible en: https://www.nejm. org/ doi/pdf/10.1056/NEJMoa0908821?articleTool$\mathrm{s}=$ true

25. Delgado C, Yepes L, Paz F, Oliveros L, Palomino Y, Sánchez $\mathrm{M}$, et al. Pancreatitis necrotizante infectada: resultados en una cohorte de baja mortalidad. Revista Colombiana de Cirugía 2006;21:240-59. 